\title{
Adult but not aged C57BL/ 6 male mice are capable of using geometry for orientation
}

\author{
Laetitia Fellini, Melitta Schachner, and Fabio Morellini ${ }^{1}$ \\ Zentrum für Molekulare Neurobiologie, Universität Hamburg, D-20251 Hamburg, Germany
}

\begin{abstract}
Geometry, e.g., the shape of the environment, can be used by numerous animal species to orientate, but data concerning the mouse are lacking. We addressed the question of whether mice are capable of using geometry for navigating. To test whether aging could affect searching strategies, we compared adult (3- to 5-mo old) and aged (20to 21-mo old) C57BL/6 male mice. We established a water maze task in which spatial information is provided by one landmark proximal to the target (featural information) and by the rectangular shape of the maze (geometric information). By means of probe trials in which we manipulated the presence of these two kinds of information, we show that adult mice can use both geometry and landmark to orientate. By contrast, aged mice do not use geometry and rely exclusively on the landmark to locate the platform. This study provides the first evidence that mice are capable of using geometric information for orientation and that this ability declines in aged animals.
\end{abstract}

Place learning is one of the most common approaches to study mechanisms of memory in animals. Place learning assumes that an invisible target can be found by using allothetic navigation, namely by relying on the diverse information (visual, auditory, and olfactory cues) emerging from the milieu. Thus, specific features in the environment (featural information) can be used as landmarks to orientate. In addition to featural information, the shape of the environment (that is, geometry) can be important information used during allothetic navigation. The studies of Cheng (1986) and Margules and Gallistel (1988) were the first to demonstrate that rats can process geometry and even use it preferentially as compared with landmarks to orientate themselves in an enclosed environment. These studies have been followed by others showing that not only rats (Sakamoto and Okaichi 1996; Ramos 2000; Pearce et al. 2001; McGregor et al. 2004) but also fish (Sovrano et al. 2002, 2003), birds (Vallortigara et al. 1990; Kelly et al. 1998; Vargas et al. 2004), and monkeys (Gouteux et al. 2001) are capable of relying on geometry to orientate.

Various species have been studied for their ability to navigate by means of geometric information, but there are no data concerning the mouse, although it has become an important species in cognitive neuroscience due to the generation of transgenic lines. As pointed out by Frick et al. (2000b), "mice are not little rats," and differences have been found between the two species, notably in terms of the searching strategies used to solve the water maze task (Frick et al. 2000b; Whishaw et al. 2001). Therefore, information acquired from the rat cannot be applied to the mouse without previous experimental evidence.

Similar to aged humans (Perlmutter et al. 1981; Sharps and Gollin 1987; Uttl and Graf 1993), aged mice exhibit memory deficits in spatial learning tasks (Lamberty and Gower 1993; Bach et al. 1999; Frick et al. 2000a). However, other studies showed that place learning is unaffected or only partially altered in aged mice and rats (Rapp et al. 1987; Nicolle et al. 2003), for instance when spatial information provided to animals is simplified (Lamberty and Gower 1991). These observations suggest that aging affects some but not all cognitive abilities required in a water maze task.

This study aims to test whether mice are capable of using

\footnotetext{
'Corresponding author.

E-mail fmorell@zmnh.uni-hamburg.de; fax +49-40-428036032

Article published online before print. Article and publication date are at http:// www.learnmem.org/cgi/doi/10.1101/lm.259206.
}

geometry to orientate and whether aging affects this ability. We designed a water maze task in which spatial information is controlled and modifiable so that, depending on the task conditions, mice are differentially exposed to featural and/or geometric information.

\section{Results}

\section{Acquisition}

Throughout the learning phase, aged mice showed slower swimming velocity than adult mice (Fig. 1A) as indicated by the significant effect of the factor age $\left(F_{1,52}=94.09 ; P<0.001\right)$. Since a slower swimming velocity can lead to higher escape latencies independently of cognitive abilities, distance covered was used to evaluate learning success. There was no effect of age nor of the interaction between age and session on distance covered during acquisition, whereas there was an effect of the factor session $\left(F_{5,260}=14.75 ; P<0.001\right)$ indicating that both aged and adult mice improved their performance by decreasing over consecutive training days the path length to reach the platform (Fig. 1B). Post hoc analyses indicated that distance covered on day 3 did not differ from distances covered during the last days of training, showing that the performance of aged and adult mice quickly reached the asymptotic level. As performances were stable during the last four learning days in both groups, we assumed that aged and adult mice had learned the task on day 6 of training. All mice showed neither floating, thigmotaxis, nor jumps out of the water throughout the experiment.

\section{First probe trial}

During the first probe trial, performed under the same conditions used during training (rectangular maze with one landmark on the short wall nearby the platform, see scheme in Fig. 2A), both aged and adult mice searched in the proximity of the platform position. To test spatial preference for the platform location, we compared the number of entries and time spent in the zone corresponding with the platform position ("T" in Fig. 2A) with three control circular zones of the size of the platform located at the three other corners of the maze (zones 1-3 in Fig. 2A). There was no effect of the factor age on number of entries and time spent in zones, whereas there was an effect of the factor zone on the number of entries $\left(F_{3,156}=69.77 ; P<0.001\right)$ and time spent in zones $\left(F_{3,156}=49.00 ; P<0.001\right)$; both groups entered more 

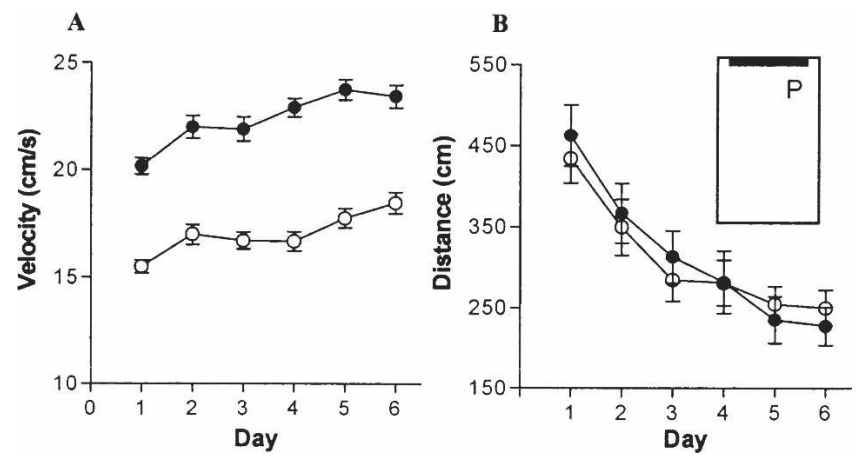

Figure 1. Despite a slower velocity, aged mice (open circles, $n=32$ ) learned the task as well as adult mice (solid circles, $n=22$ ). (A) During the $6 \mathrm{~d}$ of learning, aged mice swam more slowly than adult mice. (B) The distance needed to reach the platform decreased during the learning phase in both adult and aged mice. (Inset) The scheme of the maze. (Thick bar) Landmark position, (P) platform location.

often (Fig. 2A) and spent more time (Fig. 2B) in the target zone as compared with the other three zones. There was no effect of the interaction between age and zone on time spent in zones, whereas there was an effect on entries into zones $\left(F_{3,156}=3.20\right.$; $P<0.05)$; adult mice entered more often into the target zone than aged mice. Since no difference was observed in time spent in the target zone between the two groups, it is likely that the higher number of entries into the target zone shown by adult mice as compared with aged mice was due to differences in swimming velocity and distance covered. Indeed, as observed during learning and in all following probe trials, adult mice swam faster (adult: $22.65 \pm 0.41 \mathrm{~cm} / \mathrm{sec}$; aged: $18.39 \pm 0.36 \mathrm{~cm} / \mathrm{sec} ; P<0.001)$ and covered longer distances (adult: $1294 \pm 24.38 \mathrm{~cm}$; aged: $1043 \pm 21.90$ $\mathrm{cm} ; P<0.001)$ than aged mice. They thus increased their chance to cross the target platform as compared with aged mice. The evidence that aged and adult mice equally preferred the target zone was confirmed by the analysis of the two preference indices, measures that are independent of distance covered and swimming velocity. There was no difference between aged and adult mice for the indices calculated for entries and time. Both indices were higher than chance level in the two groups, indicating that adult and aged mice preferentially searched the platform at its correct location (Fig. 2C). This first probe trial was also used to select, for the further trials, those mice that displayed high spatial selectivity for the platform by reaching the criterion that we had determined (i.e., mice that spent at least twice as much time in the target platform as compared with the second preferred platform). Less than $50 \%$ of the aged mice reached our criterion (15 mice out of 32 ) versus nearly $70 \%$ of the adult animals (15 mice among 22) ( $\chi^{2}$ test: $P=0.122$; Fisher exact probability test: $P=0.166)$. No difference was detected between the selected aged and adult mice in their performance in this first probe trial (Fig. 2D,E,F) for any of the parameters analyzed besides mean velocity (adult: $22.93 \pm 0.52 \mathrm{~cm} / \mathrm{sec}$; aged: $17.67 \pm 0.58 \mathrm{~cm} / \mathrm{sec}$ ) and distance covered (adult: $1310 \pm 31.64 \mathrm{~cm}$; aged: $988.3 \pm 34.58 \mathrm{~cm}$ ), which showed higher values in the selected adult mice as compared with the selected aged mice $(P<0.001$ for both parameters). Interestingly, adult mice spent more time in a corridor linking the target zone and the diagonally opposite one (" $\mathrm{T}$ " and " 2 " in scheme in Fig. 2A) as compared with aged mice (adult: $43.22 \pm 2.94 \%$; aged: $33.93 \pm 2.38 \% ; P<0.05)$. In contrast, the two groups spent the same amount of time in the corridor linking the two incorrect zones (" 1 " and " 3 " in scheme in Fig. 2A) (adult: $30.00 \pm 1.07 \%$; aged: $25.16 \pm 2.16 \%$ ). The qualitative observation of the swimming paths of the selected adult and selected aged mice is in agreement with this result; in fact, in addition to a clear preference for the target zone, adult mice swam along the line connecting the target zone and its diagonally opposite one (Fig. 3A). In contrast, the swimming path of the aged group was focused in the area proximal to the target and less on the diagonal between the two geometrically correct zones (Fig. 3B). Both these quantitative and qualitative observations suggested that adult mice, but not the aged ones, orientated themselves using not only the landmark but also the shape of the maze during the first probe trial.

\section{Geometry probe trial}

The "geometry probe trial" was performed to test the ability of mice to use the shape of the maze to locate the platform. In this probe trial, two pairs of corners can be distinguished from each
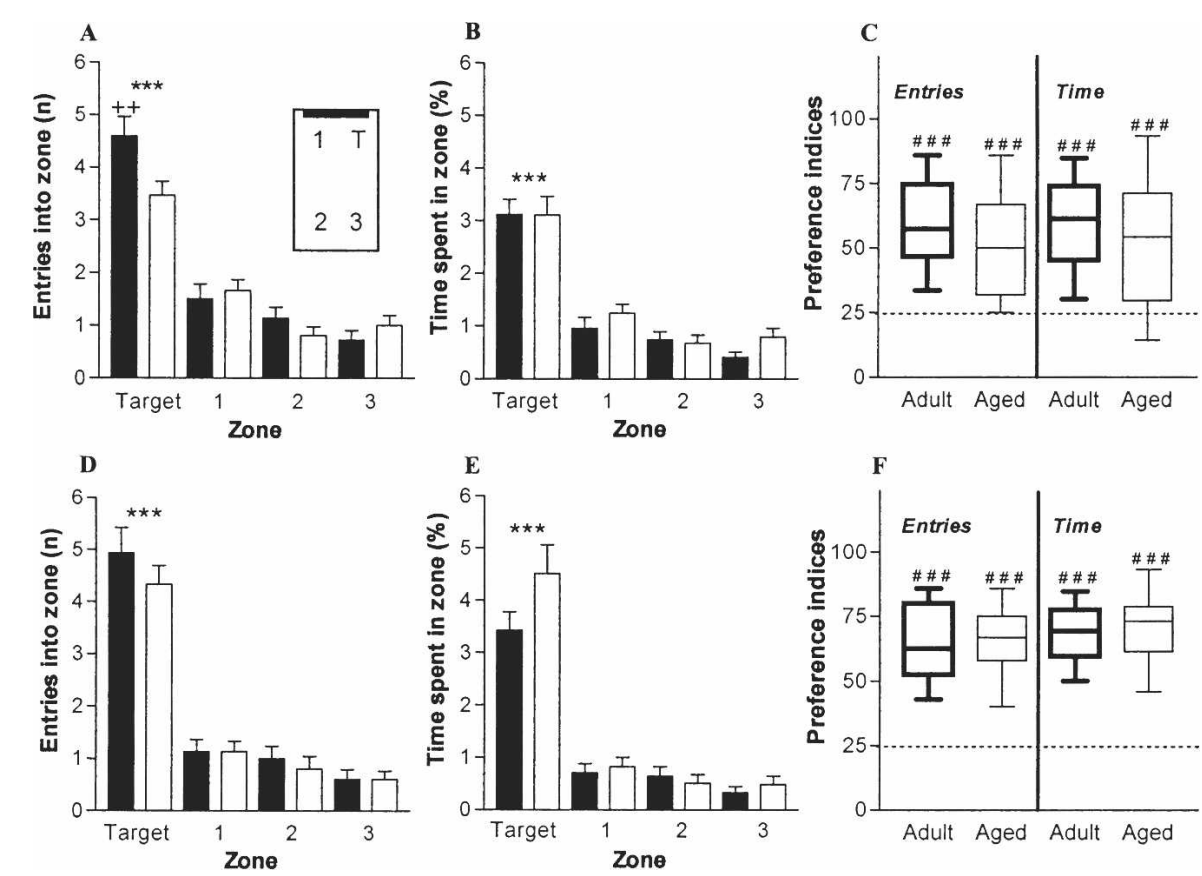

Figure 2. During the first probe trial, adult (solid bars in $A, B, D, E$; thick box plots in $C, F$ ) and aged (open bars in $A, B, D, E$; thin box plots in $C, F$ ) mice searched the platform preferentially at its correct location. $(A$, inset) The scheme of the maze. (Thick bar) Landmark position, $(T)$ target zone, $(1,2,3)$ control zones (incorrect). (A,B,C) Performance of all adults $(n=22)$ and aged $(n=32)$ mice. $(D, E, F)$ Performance of selected adult $(n=15)$ and aged $(n=15)$ mice. These mice were selected for undergoing the next probe trials. Number of entries into and time spent in zones are represented with means plus S.E.M. The preference indices are shown with standard box plots. ${ }^{* * *} P<0.001$, difference between the target zone and the other zones within both groups (Newman-Keuls post hoc after a significant effect of zone); $++P<0.01$, as compared with the aged group within the same zone (Newman-Keuls post hoc after a significant effect of the interaction between group and zone); $\# \# \#>0.001$, as compared with chance level (25\%) (Wilcoxon signed-rank test). 
other using the geometric properties of the maze, since the corners connected by one diagonal of the rectangular maze are identical to each other in terms of geometric properties. Therefore, the corner in which the platform was located during training and the diagonally opposite corner are both considered correct during the geometry probe trial ("T" in Fig. 4A), whereas the other
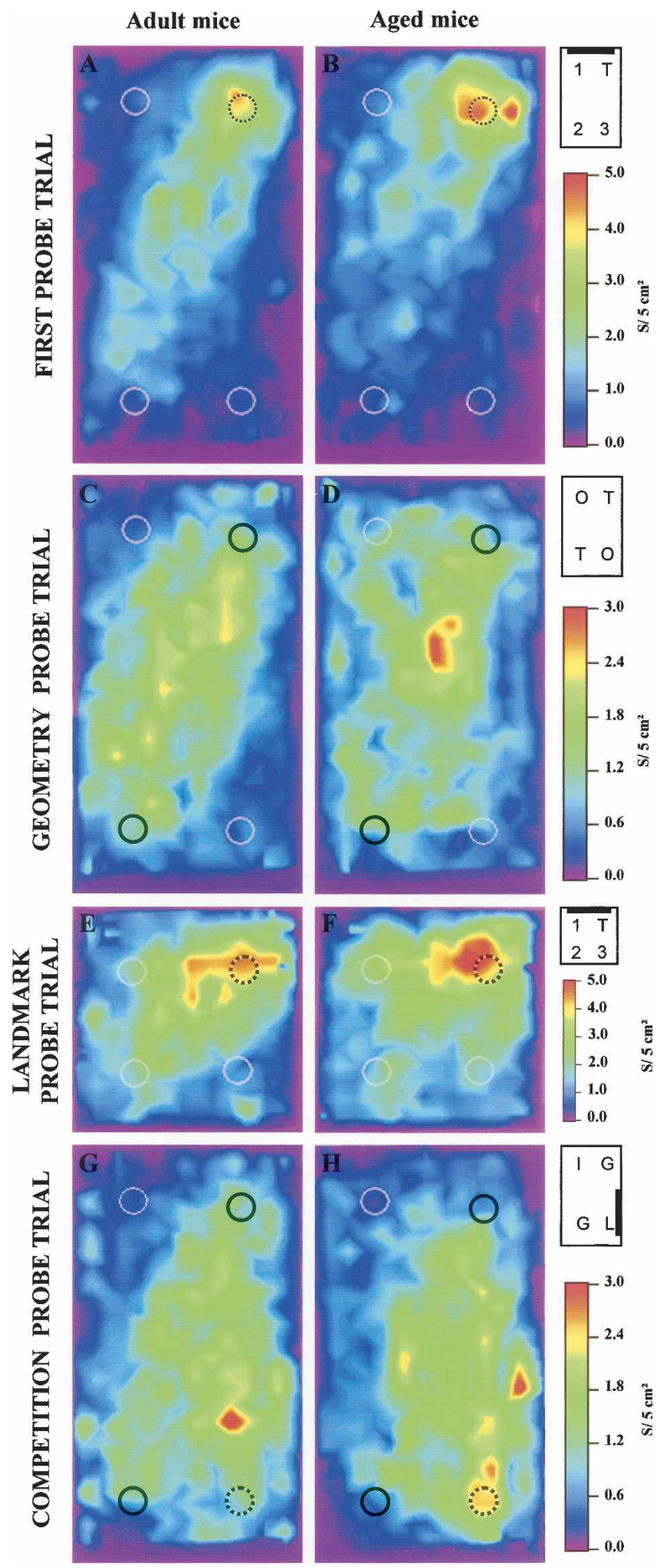

two corners are considered incorrect ("O" in Fig. 4A). Adult mice, but not the aged ones, showed a preference for the two geometrically correct target zones versus the two incorrect opposite zones (the absolute values for the two target corners and the two opposite corners, respectively, were added up and used for statistical analysis and graphical representation of entries into zones, time in zones, and preference indices; Fig. 4). There was an effect of the interaction between age and zone on number of entries into zones $\left(F_{1,28}=8.05 ; P<0.01\right)$ and a tendency on time spent in zones $\left(F_{1,28}=2.72 ; P=0.11\right)$ : While aged mice did not show any difference between entries made and time spent in the target zones as compared with the opposite zones, adult mice entered more often into and spent more time in the target zones than in the opposite ones. In addition, adult mice entered into the target zones more often than aged mice; conversely, they spent less time and tended to enter less often $(P=0.06)$ into the opposite zones as compared with aged mice (Fig. 4A,B). Since aged mice swam more slowly (adult: $22.22 \pm 0.52 \mathrm{~cm} / \mathrm{sec}$; aged: $16.73 \pm 0.66 \mathrm{~cm} / \mathrm{sec} ; P<0.001)$ and covered less distance (adult: $1265 \pm 31.63 \mathrm{~cm}$; aged: $940.80 \pm 39.33 \mathrm{~cm} ; P<0.001)$ as compared with the adult ones, the results described above could be misleading. Indeed, due to their slower velocity, aged mice had less chance to enter into and to spend time in the correct areas as compared with the adult mice. However, the comparisons between preference indices, which are independent of swimming velocity and distance covered, confirmed the differences observed between groups, as preference indices for entries and time in target zones were higher for adult mice than for aged mice. Moreover, the indices for entries and time in target zones of the adult group, but not of the aged group, were higher than chance level (Fig. 4C). Thigmotaxis and floating were not observed in the two groups. The better ability of adult mice to use geometric information as compared with aged mice was also shown by the spatial histograms representing the swimming paths (Fig. 3C,D). Interestingly, adult mice seemed to swim mostly along the diagonal connecting the two correct corners.

\section{Landmark probe trial}

Apart from the fact that the aged group swam more slowly (adult: $20.64 \pm 0.55 \mathrm{~cm} / \mathrm{sec}$; aged: $15.90 \pm 0.50 \mathrm{~cm} / \mathrm{sec} ; P<0.001)$ and covered less distance than adult mice (adult: $1142 \pm 34.21 \mathrm{~cm}$; aged: $882.3 \pm 29.81 \mathrm{~cm} ; P<0.001$ ), no difference between groups was found in the "landmark probe trial," and both groups explored preferentially the correct target zone (Figs. 3E,F, 5). There was no effect of age on entries into zones, but a tendency for an effect on time spent in zones $\left(F_{1,28}=4.05 ; P=0.054\right)$ : Aged mice spent in general more time in the four zones than adult mice. A significant effect of the factor zone was found on entries into zones $\left(F_{3,84}=20.91 ; P<0.001\right)$ and time spent in zones $\left(F_{3,84}=18.57 ; P<0.001\right)$ : Both aged and adult mice entered more often into (Fig. 5A) and spent more time in (Fig. 5B) the target

Figure 3. Spatial histograms of the mouse locations during the probe trials. Schemes of the maze and rainbow scales are shown on the right side for each probe trial. $(A, C, E, G)$ Adult group; $(B, D, F, H)$ aged group. $(A, B)$ "First probe trial." The target is depicted with a thin dotted circle on the histograms and " $T$ " in the corresponding scheme. $(C, D)$ "Geometry probe trial." The two target zones are depicted with black line circles on histogram and " $\mathrm{T}$ " in the corresponding scheme. $(E, F)$ "Landmark probe trial." The target location is depicted with a thick dotted circle and " $T$ " in the corresponding scheme. $(A-F)$ The other zones (incorrect) are depicted with a thin white circle on the histograms and "O", " 1 ", " 2, " and " 3 " in the corresponding schemes. $(G, H)$ "Competition probe trial." Geometry zones (black line circles, histogram; " $\mathrm{G}$ " in the corresponding scheme); landmark zone (thick dotted circle, histogram; " $\mathrm{L}$ " in the corresponding scheme); incorrect zone (thin white circle, histogram; "I" in the corresponding scheme). 

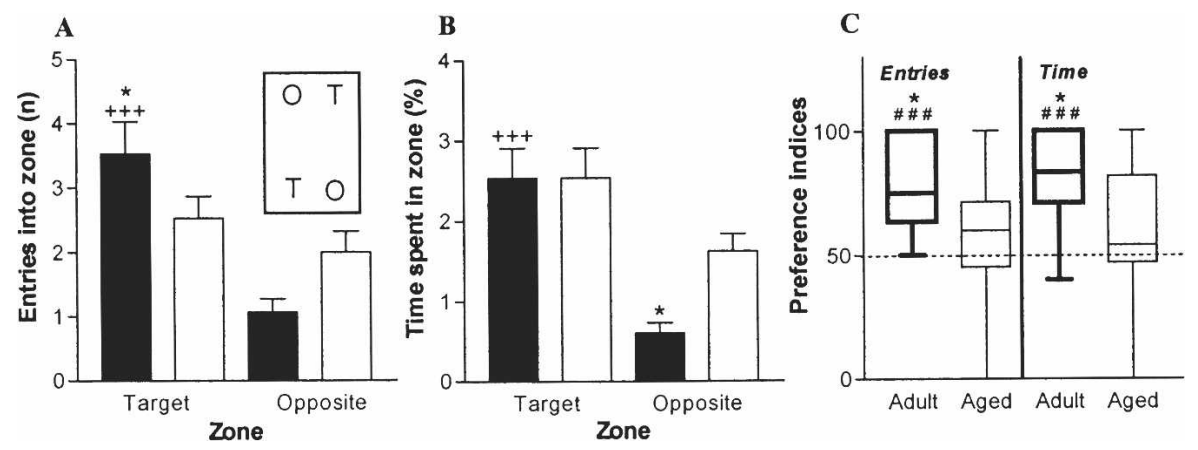

Figure 4. During the "geometry probe trial," adult mice (solid bars in $A, B$; thick box plots in $C$ ) used geometry to find the platform, whereas aged mice (open bars in $A, B$; thin box plots in $C$ ) could not orientate themselves on the basis of this sole information. ( $A$, inset) A scheme of the maze. (T) Target zones, $(\mathrm{O})$ opposite zones (incorrect). Entries into and time spent in the target and opposite zones were obtained by adding up values of the two target zones and the two opposite zones for each mouse. Number of entries into and time spent in zones are represented with means plus S.E.M. The preference indices are shown with standard box plots. $+++P<0.001$, difference between the target zone and the opposite zones within the adult group (Newman-Keuls post hoc after a significant effect of zone); $(A, B){ }^{*} P<0.05$, as compared with the aged group within the same zone (Newman-Keuls post hoc after a significant effect of the interaction between group and zone); $(C)^{*} P<0.05$ as compared with the aged group (Mann Whitney U-test); \#\#\# $<0.001$, as compared with chance level (50\%) (Wilcoxon signed-rank test).

zone as compared with the other three zones. No effect of the interaction between age and platform was detected for any parameter. The analysis of preference indices did not reveal any difference between aged and adult mice, and these indices were higher than chance level in both groups (Fig. 5C).

\section{Fourth probe trial}

The results from the fourth probe trial, which aimed to check whether mice were still motivated to find the platform and focused their search at the correct location after the "landmark" and "geometry" probe trials, replicated those obtained in the first probe trial (data not shown). Both groups preferentially searched the platform at its correct location as indicated by number of entries into and time spent in zones and by the corresponding preference indices. There was no difference between aged and adult mice, with the exception that adult mice swam faster and covered longer distances than aged mice.

\section{Competition probe trial}

During the "competition probe trial," the landmark was located on the right side of one of the long walls, instead of on one of the short walls as during acquisition (see scheme in Fig. $6 \mathrm{~A})$. Thus, if featural information, namely the landmark, was used to locate the platform, then mice would have searched in the "landmark" zone (L). If geometric information, i.e., the rectangular shape of the maze, was used, then mice would have searched in the "geometry" zones $(\mathrm{G})$. (The mean of the two "geometry" zones was used for statistical analysis and graphical representation of entries into zone, time in zone, and preference indices.) The remaining zone (I) was incorrect regarding information provided either by geometry or landmark. As during the previous probe trials, neither thigmotaxis nor floating was observed, indicating that aged and adult mice actively searched the platform. De-
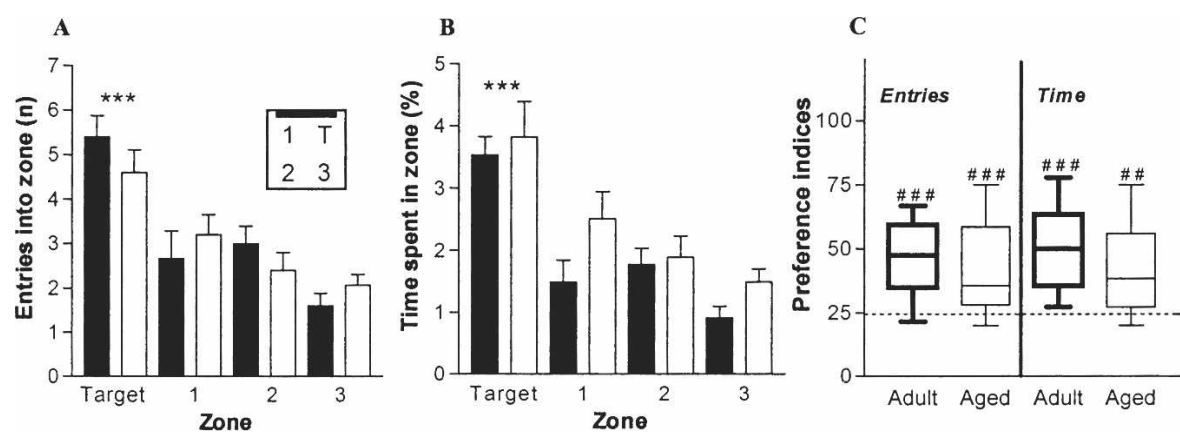

Figure 5. Adult (solid bars in $A, B$; thick box plots in $C$ ) and aged (open bars in $A, B$; thin box plots in $C)$ mice could orientate themselves and found the platform when only the featural information was available. ( $A$, inset) A scheme of the maze. (Thick bar) Landmark position, (T) target zone, $(1,2,3)$ other (incorrect) zones. Number of entries into and time spent in zones are represented by means plus S.E.M. The preference indices are shown in standard box plots. ${ }^{* *} P<0.001$, difference between the target zone and the other zones within both groups (Newman-Keuls post hoc after a significant effect on zones); $\# \# P<0.01$, \#\#\#P<0.001, as compared with chance level (25\%) (Wilcoxon signed-rank test). spite a slower velocity (adult: $21.73 \pm 0.65 \mathrm{~cm} / \mathrm{sec} ;$ aged: $16.85 \pm 0.53 \mathrm{~cm} / \mathrm{sec} ; P<0.001)$ and $1219 \pm 41.05 \mathrm{~cm}$; aged: $950.10+31.85$ $\mathrm{cm} ; P<0.001)$ in the aged group as compared with the adult group, there was no effect of age on either entries into zones effect of the interaction between age and zone on entries $\left(F_{2,56}=4.63 ; P<0.05\right)$ and on time $\left(F_{2,56}=7.92 ; P<0.001\right)$ : Aged mice entered more often into and than in the geometry or incorrect zones, while there was no difference between the geometry and incorrect zones, indicating that aged mice preferentially used the landmark to navigate (Fig. 6A,B). In contrast, adult mice searched the platmasing both geometry and landin the geometry zones as much as in the landmark ones and entered into the incorrect zone less frequently than into the geometry zone and, although not significantly, into the landmark zone $(P<0.07)$. Adult mice also tended to spend less time in the incorrect zone as compared with the geometry zones and spent less time in the landmark zone as compared with aged mice (Fig. 6A,B). To further test age-dependent differences in the preference for the geometry and landmark zones, we compared preference indices for entries and time calculated for landmark, There was no effect of age but a tendency for an effect of the interaction between age and zone on the preference index for entries $\left(F_{2,56}=1.94 ; P=0.15\right)$ and a significant effect on the index for time $\left(F_{2,56}=3.97 ; P<0.05\right)$. Post hoc analysis revealed the landmark zone were higher than those for the geometry and incorrect zones. Moreover, the preference indices for entries and time for the landmark zone were higher than chance level, whereas those for the geometry zones were equivalent to chance level, confirming that aged mice preferentially searched in the landmark zone but not in the geometry zones (Fig. 6C,D). In the 

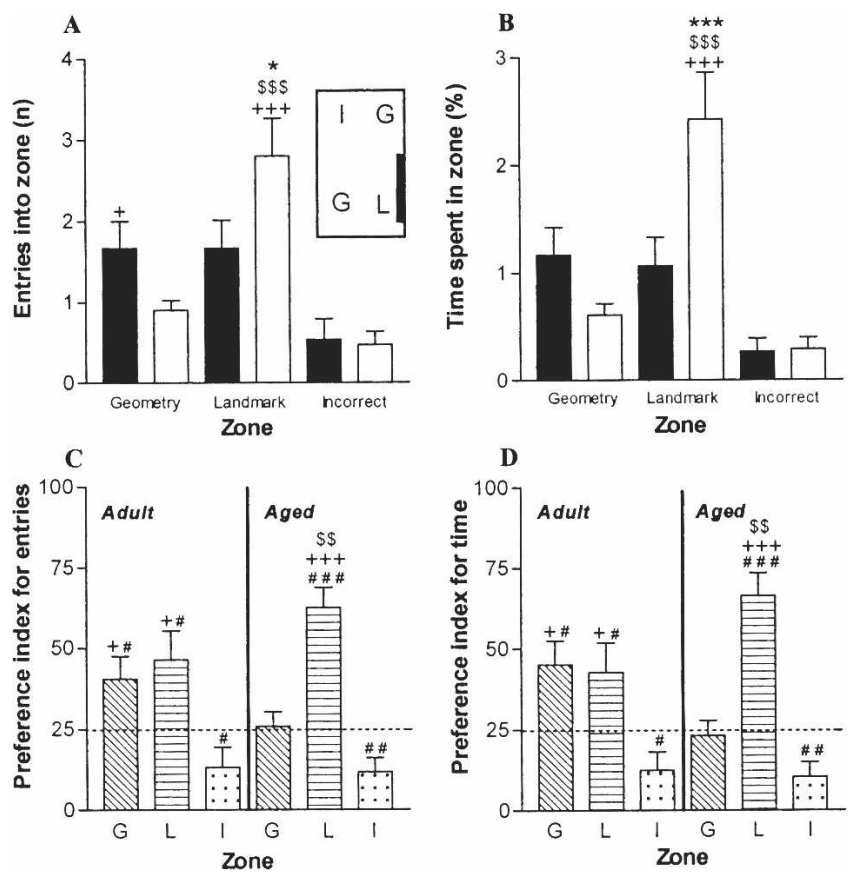

Figure 6. During the "competition probe trial," adult mice (solid bars in $A, B$ and left side of $C, D$ ) flexibly relied either on landmark or on geometry, while aged mice (open bars in $A, B$ and right side of $C, D$ ) followed only the landmark indication. $(A$, inset) $A$ scheme of the maze. (Thick bar) Landmark position, (G) zones designed by geometry, $(\mathrm{L})$ zone indicated by landmark, (I) zone that was incorrect regarding landmark and geometric information. Values represent means plus S.E.M. $+P<0.05$, $+++P<0.001$, as compared with the incorrect zone within each group; $\$ \$ P<0.01, \$ \$ \$ P<0.001$, as compared with the geometry zones within the aged group; ${ }^{*} P<0.05,{ }^{* * *} P<0.001$, as compared with the adult group within the same zone (Newman-Keuls post hoc after a significant effect of the interaction between group and zone); $\# P<0.05$, $\# \# P<0.01$, \#\#\#P<0.001, as compared with chance level $(25 \%)$ (Wilcoxon signed-rank test).

adult group there was no difference between preference indices for entries and time in geometry zones and those for the landmark zone; in addition, indices for geometry and landmark zones were higher than those for incorrect zone and than chance level (Fig. 6C,D). We also analyzed all parameters for the three 20-sec intervals to test whether mice changed searching strategies during the probe trial. The same results as those described previously were obtained, and no effect of time interval was observed, indicating that the strategy used by each group was stable throughout the probe trial (data not shown). Finally, we performed a qualitative analysis of individual performances of both groups (Table 1). Interestingly, in the adult group, three kinds of searching strategies have been noticed: Some mice preferentially searched in the landmark zone, others in the geometry zones, and others switched between the landmark and geometry zones. In contrast, no aged mouse showed a preference exclusively for the geometry zones, and only two animals used both geometric and landmark information, while the majority of the aged group followed the information provided by the landmark.

\section{Control probe trial}

We performed a final probe trial to test whether uncontrolled cues could have influenced the spatial selectivity of the mice. In the absence of landmark and of geometric information, mice did not show any preference for any of the four corners, indicating that no uncontrolled cue had influenced the searching strategies throughout the experiment. There was an effect of the factor age on number of entries $\left(F_{1,28}=4.32 ; P<0.05\right)$ (Fig. $\left.7 \mathrm{~A}\right)$ but not on time spent in zones (Fig. 7B): Adult mice crossed the four zones more often than the aged, probably as a consequence of their higher swimming velocity (adult: $19.81 \pm 0.65 \mathrm{~cm} / \mathrm{sec}$; aged: $14.99 \pm 0.44 \mathrm{~cm} / \mathrm{sec} ; P<0.001)$ and longer distance covered (adult: $1084 \pm 40.61 \mathrm{~cm}$; aged: $826.50 \pm 25.22 \mathrm{~cm}$ ). However, neither effect of zone nor effect of the interaction between group and zone was found for any parameter.

\section{Discussion}

This study was designed to assess whether mice are capable of using geometry, in this case the shape of an enclosed environment, to orientate themselves and, if so, whether they can interchangeably use geometric and featural information, namely a prominent landmark. In addition, we wanted to test whether the use of geometric and featural information changes with aging. We showed that adult mice flexibly use geometric and featural information to navigate and do not display preferred strategy, while aged mice do not use geometry and rely on featural information.

\section{Mice process geometry and use it for navigating}

Our data demonstrate that adult mice can use the shape of the environment to find a hidden goal, indicating that they detect

Table 1. Individual performance of adult and aged mice in the competition probe trial

\begin{tabular}{|c|c|c|c|c|c|c|}
\hline \multirow[b]{2}{*}{ Adult mice } & \multicolumn{3}{|c|}{ Entries into zone (n) } & \multicolumn{3}{|c|}{ Time in zone $(\%)^{d}$} \\
\hline & $G^{a}$ & $L^{b}$ & $\mathrm{I}^{\mathrm{C}}$ & G & $\mathbf{L}$ & I \\
\hline 1 & 4 & 1 & 0 & 2.8 & 0.7 & 0.0 \\
\hline 2 & 4.5 & 4 & 0 & 3.8 & 2.7 & 0.0 \\
\hline 3 & 1 & 0 & 3 & 0.7 & 0.0 & 1.3 \\
\hline 4 & 0.5 & 2 & 0 & 0.3 & 2.0 & 0.0 \\
\hline 5 & 1.5 & 4 & 0 & 1.0 & 3.0 & 0.0 \\
\hline 6 & 1 & 1 & 1 & 0.8 & 0.3 & 0.7 \\
\hline 7 & 2 & 1 & 2 & 1.0 & 0.3 & 1.0 \\
\hline 8 & 1 & 3 & 0 & 1.0 & 1.3 & 0.0 \\
\hline 9 & 2 & 0 & 2 & 1.5 & 0.0 & 1.0 \\
\hline 10 & 0 & 3 & 0 & 0.0 & 2.3 & 0.0 \\
\hline 11 & 1.5 & 1 & 0 & 1.0 & 0.3 & 0.0 \\
\hline 12 & 0 & 1 & 0 & 0.0 & 0.7 & 0.0 \\
\hline 13 & 2 & 2 & 0 & 1.0 & 1.0 & 0.0 \\
\hline 14 & 2.5 & 0 & 0 & 1.3 & 0.0 & 0.0 \\
\hline \multirow[t]{2}{*}{15} & 1.5 & 2 & 0 & 1.2 & 1.3 & 0.0 \\
\hline & \multicolumn{3}{|c|}{ Entries into zone (n) } & \multicolumn{3}{|c|}{ Time in zone $(\%)^{d}$} \\
\hline Aged mice & $\mathrm{G}^{\mathrm{a}}$ & $L^{b}$ & $I^{c}$ & G & $\mathbf{L}$ & $I$ \\
\hline 16 & 1.5 & 6 & 0 & 0.8 & 4.7 & 0.0 \\
\hline 17 & 0.5 & 3 & 1 & 0.7 & 2.3 & 0.7 \\
\hline 18 & 0.5 & 1 & 0 & 0.5 & 0.7 & 0.0 \\
\hline 19 & 0.5 & 5 & 0 & 0.3 & 3.7 & 0.0 \\
\hline 20 & 1 & 1 & 0 & 0.3 & 0.7 & 0.0 \\
\hline 21 & 1.5 & 3 & 2 & 1.0 & 3.3 & 1.3 \\
\hline 22 & 1 & 1 & 1 & 0.7 & 0.3 & 0.7 \\
\hline 23 & 0.5 & 4 & 0 & 0.3 & 3.7 & 0.0 \\
\hline 24 & 0.5 & 2 & 0 & 0.2 & 2.0 & 0.0 \\
\hline 25 & 1.5 & 4 & 0 & 1.2 & 3.0 & 0.0 \\
\hline 26 & 1 & 0 & 1 & 0.5 & 0.0 & 0.7 \\
\hline 27 & 0 & 3 & 1 & 0.0 & 3.3 & 0.3 \\
\hline 28 & 1 & 5 & 1 & 0.8 & 5.7 & 0.7 \\
\hline 29 & 1.5 & 1 & 0 & 1.5 & 1.0 & 0.0 \\
\hline 30 & 1 & 3 & 0 & 0.3 & 2.0 & 0.0 \\
\hline
\end{tabular}

${ }^{a}$ Geometry zone values correspond to the mean of number of entries into and time spent in the two geometrically correct zones.

b Landmark zone.

'Incorrect zone.

${ }^{d}$ According to chance, time spent in one zone would be $0.5 \%$. 

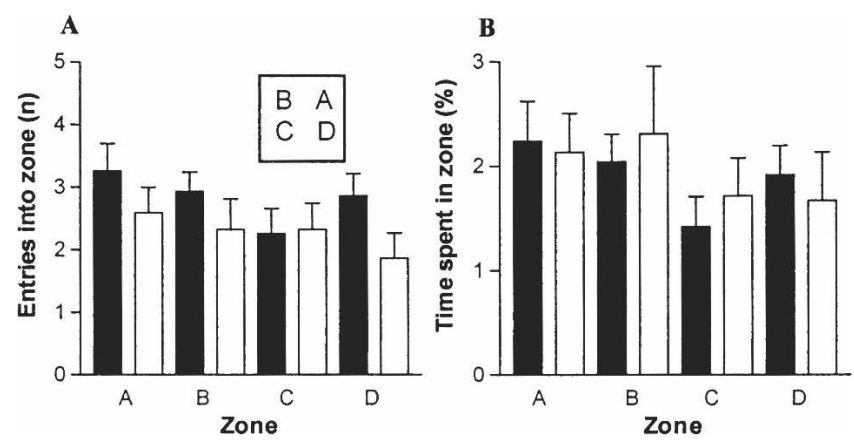

Figure 7. During the control probe trial, adult (solid bars) and aged (open bars) mice did not show any preference for one of the four possible zones, as indicated by number of entries into $(A)$ and time spent in $(B)$ zone, confirming that no extra-maze cues influenced the searching strategy of the mice. $(A$, inset) A scheme of the maze. The different zones ( $A$, $B, C, D)$ were of the same size as the platform. Values represent means plus S.E.M.

the geometric properties of the environment and use them to navigate. How this information is used is, however, unclear. Three possibilities are conceivable to interpret these observations. First, mice process the global shape of the environment (i.e., "this environment is a rectangle"). Second, they could rely only on the local properties of the environment (i.e., "the platform is situated in the corner that presents a long wall on the right hand"). Or, finally, they could use a basic procedural strategy by learning to turn left when they are facing a long wall. We exclude this last possibility: Adult mice swam along the line connecting the two geometrically correct corners, suggesting that they are capable of extrapolating the diagonal of the rectangular maze by processing either the global shape of the environment or its local properties.

It is noteworthy that geometric information was acquired by mice even if it was not crucial to solve the task. In fact, during learning, the landmark provided a more precise information than geometry since it indicated without any ambiguity the platform position among the four possible corners; in contrast, geometric information led to two possibilities, namely the target corner and its diagonally opposite one. These results add further to those obtained in other species and provide evidence for the first time that mice perceive and are capable of using geometry for orientation. In addition, we show that in mice, as in other species such as fish (Sovrano et al. 2003), chicks (Vallortigara et al. 1990), pigeons (Vargas et al. 2004), and rats (Pearce et al. 2001; Wall et al. 2004), the presence of a landmark does not prevent animals from acquiring geometric information. From an ecological point of view, it is likely that geometry is more important than landmarks: As argued by Sovrano et al. (2002), in wildlife the large-scale shape of the environment provides stable and thus reliable information to orientate, as opposed to featural information such as vegetation that undergoes seasonal changes. This could explain that mice learn to use geometry to orientate also when it is superfluous, as in our study in which the platform was reliably indicated by a prominent landmark.

Interestingly, in a conflictual situation in which geometry and landmark provide contradictory indications, mice rely flexibly on one or the other information. As shown by the analysis of the individual performance during the "competition probe trial," some mice rely preferentially on the landmark while others use geometry, and some switch between the two. These results are in contrast with those demonstrating that a preference emerges in conflictual situations as observed in pigeons (Vargas et al. 2004; but see also Kelly et al. 1998), chicks (Vallortigara et al. 1990; Tommasi et al. 2003), and monkeys (Gouteux et al. 2001). It has been proposed that the size of the apparatus could be important for choosing between geometric and landmark information, as observed in young children who preferentially use landmarks in large environments and geometry in small ones (Learmonth et al. 2002). Sovrano et al. (2005) and Vallortigara et al. (2005) have also suggested that this phenomenon could occur in fish and chicks. In our case, it could be argued that our maze has intermediate dimensions, preventing a clear preference for geometry or landmark information in adult mice.

\section{Aging impairs the flexible use of geometry and landmark for navigating}

No difference could be detected between adult and aged mice during learning and the first probe trial performed in the presence of congruent landmark and geometric information. Interestingly, the difference between the two groups became apparent when we manipulated spatial information (landmark and/or geometry). In fact, aged mice were impaired when only geometry was accessible but not when the landmark was present. This inability to use geometry for navigation was confirmed in the "competition probe trial," in which aged mice followed only the information indicated by the landmark. It has been shown previously that cognitive decline differs among individuals (Bach et al. 1999; Schulz et al. 2002; Nicholson et al. 2004; but see also Frick et al. 1995). In our experiment we observed few aged mice performing as well as adult ones in the "geometry probe trial." Nevertheless, the majority of the aged group was not able to use geometry to navigate, an inability that is unlikely due to lower motivation, swimming impairments, or sight problems, since these mice performed well when the landmark was available.

It is possible that the impairment of aged mice is due to their altered stress response, as it is well known that stress can affect the learning rate (for reviews, see Bowman 2005; Shors 2006). Indeed, aged animals display a dysregulation of the hypophysispituitary-adrenal axis (Dalm et al. 2005); notably, they show longer responses to stressors as compared with adult animals (Bizon et al. 2001). However, the stressfulness of an experimental paradigm also depends on the control that animals have on the stressor. For instance, Kavushansky et al. (2006) showed that a forced swim test, but not a standard Morris water maze task, increases the plasma level of corticosterone in rats as compared with home-cage control rats. Thus, if being placed in a water tank can be stressful for mice, the possibility of finding a platform strongly reduces the stressfulness of the task. Indeed, we did not observe any stress-related behavior in the two groups during all experiments, such as floating or thigmotaxis. On the contrary, aged and adult mice actively searched for the platform and stood on it quietly until they were removed from the apparatus.

We cannot exclude the possibility that all aged mice would have learned to use geometry with extensive training, meaning that age-dependent cognitive decline prevents only a fast acquisition of geometric information. Moreover, it is possible that in the presence of both landmark and geometry, aged mice simply rely directly on the easiest information for them (i.e., the landmark). Therefore, it is conceivable that geometry is overshadowed or blocked by featural information specifically in aged but not in adult mice. Indeed, it has been reported that place learning is blocked in aged rats previously trained with a cued platform (Rapp et al. 1987).

Electrophysiological and lesion studies demonstrated that the hippocampus plays a key role in geometry-based navigation (Muller and Kubie 1987; Sakamoto and Okaichi 1996; Lever et al. 2002; Tommasi et al. 2003; McGregor et al. 2004; Vargas et al. 2004). More specifically, the hippocampus could be important for processing local metric properties such as angles and dis- 
tances to create a cognitive representation of the environment's shape. Indeed, it has been demonstrated that hippocampal place cells are sensitive to the length of a wall and to the distance from a wall (O'Keefe and Burgess 1996) and that the hippocampus is essential for processing metric properties of the environment (Sakamoto and Okaichi 1997). However, the hippocampus is not the only structure implicated in this cognitive process, since it has been shown that hippocampal lesions do not prevent cells of other structures (i.e., the postsubiculum and the anterior thalamic nucleus) to be sensitive to changes in the environmental shape (Golob and Taube 1997). We tend to consider that the impaired ability of aged mice to navigate by means of geometry is related to age-dependent hippocampal alterations (for review, see Rosenzweig and Barnes 2003). Notably, aged mice could be incapable of processing metric properties of the environment such as the length of the walls, which is crucial information for defining the shape of the rectangular maze. It is also conceivable that, although being capable to detect and process geometric information, aged mice are not able to use this information to navigate. In any case, it is possible that an impaired function of the hippocampus is compensated for by a stronger activation of other brain regions that are also involved in navigation, with the result that the particular form of navigation controlled by these structures will be predominantly used. For instance, aged mice tend to use preferentially cue-based strategies (Nicolle et al. 2003), which are mainly controlled by the striatum (Packard and McGaugh 1996; Oliveira et al. 1997; Colombo et al. 2003). It is interesting in this context that rats displaying a poorer performance in the spatial version of the Morris water-maze task show higher expression of NMDA receptors in the striatum (Nicolle et al. 1996). We therefore hypothesize that aged mice are not capable of using geometry to navigate due to an impaired hippocampal function, and that this inability is compensated for by activation of other regions, such as the striatum, that allows aged mice to navigate by means of cue-based strategies.

\section{Conclusions}

This study shows for the first time that mice are capable of detecting and using geometry during navigation, an ability that degrades with aging. Whereas adult mice can face environmental changes by relying either on geometry or landmarks to orientate, aged mice depend on featural information. More specific experiments are, however, needed to gain insights into the mechanisms underlying navigation based on geometry. Place cells recordings, lesion studies, and pharmacological studies in behaving mice could be a means to achieve this goal. Finally, the water maze task established in this study can be a sensitive tool not only to investigate spatial learning and memory processes in wild-type animals, but also to characterize cognitive abilities of mutant mice.

\section{Materials and Methods}

\section{Animals and husbandry}

C57BL/6J male mice were transferred from a pathogen-free breeding facility into a vivarium with an inverted $12 \mathrm{~h}$ light $/ 12 \mathrm{~h}$ dark cycle (lights off at 7:00 a.m.) and maintained in groups of two to five siblings under standard housing conditions $\left(23 \pm 1{ }^{\circ} \mathrm{C}, 40 \%-50 \%\right.$ humidity, food and water ad libitum). After 2 wk of acclimatization, mice underwent the experiments, which were performed between 9:00 and 15:00 in a room adjacent to the vivarium illuminated by red light. At the beginning of the experiment, 32 mice composed the aged group (20- to 21-mo old) and 22 mice composed the adult group (3- to 5-mo old).

\section{Apparatus}

A rectangular maze $(126 \times 66 \mathrm{~cm}$ and $50 \mathrm{~cm}$ high $)$ made of white polyvinylchloride easily convertible into a square $(66 \times 66 \mathrm{~cm}$ and $50 \mathrm{~cm}$ high) was used. The maze could be rotated on its central axis. A white platform (diameter $7.4 \mathrm{~cm} ; 1 \mathrm{~cm}$ below the water surface) was placed in a fixed corner with its center located $18 \mathrm{~cm}$ from the two walls. The apparatus was filled up to $9 \mathrm{~cm}$ from its bottom with water $\left(20^{\circ} \mathrm{C} \pm 1^{\circ} \mathrm{C}\right)$ made opaque by the addition of non-toxic white paint. To provide supplemental markers of the shape of the apparatus, black metal stripes $(10 \mathrm{~cm}$ high and $6 \mathrm{~cm}$ wide) were located at the four corners of the maze, $20 \mathrm{~cm}$ above the water surface. A black and white landmark (21 $\mathrm{cm}$ high and $50 \mathrm{~cm}$ wide) was placed in the middle of the short wall proximal to the platform, $8 \mathrm{~cm}$ above the water surface. This maze was circled by dark curtains hanging from the ceiling to the floor. The ceiling was covered by a white curtain with a hole in its center to allow the view from a video camera placed behind the curtain. Light was provided by four white light neon tubes placed on the floor around the swimming pool, which provided a homogeneous illumination of the maze (50 lux on the water surface). Electronic equipment for video recording and automatic tracking of the swimming paths of the mice was placed in a room adjacent to the experimental one and illuminated by dim red light. Mice were kept in this room between trials while waiting to be tested.

\section{Protocol}

Before the experiment started, mice were familiarized for $3 \mathrm{~d}$ to swim and climb onto a platform (four trials per day; 60 sec maximal duration; 10-min inter-trial interval). To avoid any interference with learning, familiarization took place in a different room and apparatus $(42.5 \times 26.5 \mathrm{~cm}$ and $15.5 \mathrm{~cm}$ high, circled by a black curtain) than that used for learning in the water maze. The position of the platform (diameter of $10 \mathrm{~cm}, 1 \mathrm{~cm}$ below the water surface) was unpredictable, since its location was randomized and we did not provide any information that could guide the animals. Thus, mice had to swim at random to escape from the water. From experimental day 1 to day 6, mice were trained in the rectangular maze described previously (see scheme in Fig. 1) with four trials per day (inter-trial interval of $10 \mathrm{~min}$ ). Four starting positions (two were situated in the middle of the long walls and two at the most distant corners to the platform) were pseudo-randomized and the apparatus was rotated by $90^{\circ}$ between trials, clockwise or counter-clockwise depending on the day, to avoid that mice could use extra-maze cues (e.g., sounds or odors) to orientate. Animals were released into the water facing the wall and were allowed $60 \mathrm{sec}$ to find the platform " $\mathrm{P}$ " in scheme in Fig. 1) and climb onto it. After staying on the platform for $30 \mathrm{sec}$, mice were returned to their home cage and kept warm under red light until the next trial. From days 7-11, mice underwent one probe trial (60 sec in duration) per day. During each probe trial, the platform was removed and mice were released from the center of the maze. To avoid extinction, mice underwent three learning trials starting $10 \mathrm{~min}$ after the probe trial under the same conditions as those used during learning on days 1-6. Depending on the day, spatial information provided during the probe trial was manipulated. The first probe trial (day 7) took place with the same spatial information available during learning (geometry and landmark information were the same as during learning, see scheme in Fig. 2) and aimed to evaluate spatial selectivity for the platform position. Only mice showing spatial selectivity for the platform position (determined by a criterion described below under "Behavioral Analysis") underwent the probe trials performed from days $8-11$. On days 8 and 9, the so-called "landmark" and "geometry" probe trials were carried out. During the "geometry probe trial," the landmark was removed while the maze had the same rectangular shape as during learning (see scheme in Fig. 4); under these conditions the geometrically correct platform position was in the two corners indicated with " $\mathrm{T}$ ". Indeed, the corners identical to each other in terms of geometric properties are those connected by one diagonal of the rectangular maze. Therefore, the corner in which the platform was located during training and the diagonally opposite corner are both considered correct during this probe trial. Conversely, during the "landmark probe trial," geometric informa- 
tion was absent since the rectangular maze was converted into a square while the landmark was still present on one of the four walls (see scheme in Fig. 5); based on the landmark, the correct position of the platform was in corner " $\mathrm{T}$ ". To avoid possible bias due to the temporal order of the two probe trials, half of the mice from each age group were tested in the "landmark probe trial" on day 8 and in the "geometry probe trial" on day 9, whereas the other half was tested in the "geometry probe trial" on day 8 and in the "landmark probe trial" on day 9. To control whether mice still had a spatial preference for the target after the "landmark" and "geometry" probe trials, a fourth probe trial was performed on day 10, which took place under the same conditions used for the probe trial on day 7. On day 11, a "competition probe trial" was performed in the rectangular maze, where both landmark and geometric information were available but provided conflictual information since the landmark was placed on one of the long walls of the maze (see scheme in Fig. 6). In fact, based only on the landmark, the platform should be searched at corner " $\mathrm{L}$ ", while the geometrically correct corners would be those indicated with "G". Finally, we performed a "control probe trial" on day 12 , where both landmark and geometric information were removed (see scheme in Fig. 7) and mice were expected to equally search in all corners.

\section{Behavioral analysis}

All trials were video recorded, and animal paths were digitalized and analyzed with the software Ethovison (Noldus). For the learning trials, velocity, distance covered, escape latency, and thigmotaxis (time spent in an 8-cm width zone that borders the wall) were calculated. For all the probe trials, we calculated number of entries into and time spent in four circular zones of size of the platform located at the previous platform location and at its virtual mirror positions in the three other corners. Distance covered, velocity, and thigmotaxis were also calculated for all probe trials. In addition, we calculated two preference indices for the target platform that are independent of swimming velocity and distance covered. One index (preference index for entries) corresponded to the number of entries into the target zone relative to the total number of entries into all zones; the second index (preference index for time) was calculated as the previous one, but using time spent in zone as the parameter. For the first probe trial, we evaluated the spatial preference of mice and selected for the further trials only those mice reaching a criterion of high spatial selectivity for the target zone; namely, mice that during the probe trial spent at least twice as much time in the target platform as compared with the second preferred platform. We calculated also for this first probe trial the time spent in a corridor linking the target zone and its diagonally opposite one. This corridor was $20 \mathrm{~cm}$ in width and did not include the two platform zones. For a qualitative analysis of the swimming paths of the two groups during the probe trials, we generated spatial histograms using the software IgorPro (WaveMetrics).

\section{Statistical analysis}

Learning trials were analyzed with a two-way ANOVA for repeated measures (having "age" as the between-groups factor and "session" as the within-groups factor), followed by NewmanKeuls post hoc analysis when appropriate. Number of entries and time spent in zones calculated during probe trials were also analyzed with a two-way ANOVA for repeated measures (having "age" as the between-groups factor and "zone" as the withingroups factor), followed by Newman-Keuls post hoc analysis when appropriate. These data are presented as means plus S.E.M. The comparison between groups of swimming velocity, distance covered, preference indices, and time spent in the corridor linking the target zone and its diagonally opposite one was performed with a Mann-Whitney U-test within each probe trial. A Wilcoxon signed-rank test was used to compare the time spent in the thigmotaxis zone and the preference indices of both groups with chance level. Data analyzed with parametric statistics (twoway ANOVA) are represented as means plus S.E.M., whereas data analyzed with non-parametric statistics (Mann-Whitney U and
Wilcoxon signed-rank tests) are represented as standard box plots.

\section{References}

Bach, M.E., Barad, M., Son, H., Zhuo, M., Lu, Y.F., Shih, R., Mansuy, I. Hawkins, R.D., and Kandel, E.R. 1999. Age-related defects in spatial memory are correlated with defects in the late phase of hippocampal long-term potentiation in vitro and are attenuated by drugs that enhance the cAMP signaling pathway. Proc. Natl. Acad. Sci. 96: $5280-5285$.

Bizon, J.L., Helm, K.A., Han, J.S., Chun, H.J., Pucilowska, J., Lund, P.K., and Gallagher, M. 2001. Hypothalamic-pituitary-adrenal axis function and corticosterone receptor expression in behaviourally characterized young and aged Long-Evans rats. Eur. J. Neurosci. 14: $1739-1751$.

Bowman, R.E. 2005. Stress-induced changes in spatial memory are sexually differentiated and vary across the lifespan. $J$. Neuroendocrinol. 17: 526-535.

Cheng, K. 1986. A purely geometric module in the rat's spatial representation. Cognition 23: 149-178.

Colombo, P.J., Brightwell, J.J., and Countryman, R.A. 2003. Cognitive strategy-specific increases in phosphorylated cAMP response element-binding protein and c-Fos in the hippocampus and dorsal striatum. J. Neurosci. 23: 3547-3554.

Dalm, S., Enthoven, L., Meijer, O.C., van der Mark, M.H., Karssen, A.M., de Kloet, E.R., and Oitzl, M.S. 2005. Age-related changes in hypothalamic-pituitary-adrenal axis activity of male C57BL/6J mice. Neuroendocrinology 81: 372-380.

Frick, K.M., Baxter, M.G., Markowska, A.L., Olton, D.S., and Price, D.L. 1995. Age-related spatial reference and working memory deficits assessed in the water maze. Neurobiol. Aging 16: 149-160.

Frick, K.M., Burlingame, L.A., Arters, J.A., and Berger-Sweeney, J. 2000a. Reference memory, anxiety and estrous cyclicity in C57BL/6NIA mice are affected by age and sex. Neuroscience 95: 293-307.

Frick, K.M., Stillner, E.T., and Berger-Sweeney, J. 2000b. Mice are not little rats: Species differences in a one-day water maze task. Neuroreport 11: 3461-3465.

Golob, E.J. and Taube, J.S. 1997. Head direction cells and episodic spatial information in rats without a hippocampus. Proc. Natl. Acad. Sci. 94: 7645-7650.

Gouteux, S., Thinus-Blanc, C., and Vauclair, J. 2001. Rhesus monkeys use geometric and nongeometric information during a reorientation task. J. Exp. Psychol. Gen. 130: 505-519.

Kavushansky, A., Vouimba, R.M., Cohen, H., and Richter-Levin, G. 2006. Activity and plasticity in the CA1, the dentate gyrus, and the amygdala following controllable vs. incontrollable water stress. Hippocampus 16: 35-42.

Kelly, D.M., Spetch, M.L., and Heth, C.D. 1998. Pigeons' (Columba livia) encoding of geometric and featural properties of a spatial environment. J. Comp. Psychol. 112: 259-269.

Lamberty, Y. and Gower, A.J. 1991. Simplifying environmental cues in a Morris-type water maze improves place learning in old NMRI mice. Behav. Neural Biol. 56: 89-100.

. 1993. Spatial processing and emotionality in aged NMRI mice: A multivariate analysis. Physiol. Behav. 54: 339-343.

Learmonth, A.E., Nadel, L., and Newcombe, N.S. 2002. Children's use of landmarks: Implications for modularity theory. Psychol. Sci. 13: $337-341$.

Lever, C., Wills, T., Cacucci, F., Burgess, N., and O'Keefe, J. 2002. Long-term plasticity in hippocampal place-cell representation of environmental geometry. Nature 416: 90-94.

Margules, G. and Gallistel, C.R. 1988. Heading in the rat: Determination by environmental shape. Anim. Learn. Behav. 16: 404-410.

McGregor, A., Hayward, A.J., Pearce, J.M., and Good, M.A. 2004. Hippocampal lesions disrupt navigation based on the shape of the environment. Behav. Neurosci. 118: 1011-1021.

Muller, R.U. and Kubie, J.L. 1987. The effects of changes in the environment on the spatial firing of hippocampal complex-spike cells. J. Neurosci. 7: 1951-1968.

Nicholson, D.A., Yoshida, R., Berry, R.W., Gallagher, M., and Geinisman, Y. 2004. Reduction in size of perforated postsynaptic densities in hippocampal axospinous synapses and age-related spatial learning impairments. J. Neurosci. 24: 7648-7653.

Nicolle, M.M., Bizon, J.L., and Gallagher, M. 1996. In vitro autoradiography of ionotropic glutamate receptors in hippocampus and striatum of aged Long-Evans rats: Relationship to spatial learning. Neuroscience 74: 741-756.

Nicolle, M.M., Prescott, S., and Bizon, J.L. 2003. Emergence of a cue strategy preference on the water maze task in aged C57B 6 SJL F1 hybrid mice. Learn. Mem. 10: 520-524.

O'Keefe, J. and Burgess, N. 1996. Geometric determinants of the place 
fields of hippocampal neurons. Nature 381: 425-428.

Oliveira, M.G., Bueno, O.F., Pomarico, A.C., and Gugliano, E.B. 1997. Strategies used by hippocampal- and caudate-putamen-lesioned rats in a learning task. Neurobiol. Learn. Mem. 68: 32-41.

Packard, M.G. and McGaugh, J.L. 1996. Inactivation of hippocampus or caudate nucleus with lidocaine differentially affects expression of place and response learning. Neurobiol. Learn. Mem. 65: 65-72.

Pearce, J.M., Ward-Robinson, J., Good, M., Fussell, C., and Aydin, A. 2001. Influence of a beacon on spatial learning based on the shape of the test environment. J. Exp. Psychol. Anim. Behav. Process. 27: 329-344.

Perlmutter, M., Metzger, R., Nezworski, T., and Miller, K. 1981. Spatial and temporal memory in 20 to 60 year olds. J. Gerontol. 36: 59-65.

Ramos, J.M. 2000. Influence of the shape of the experimental room on spatial learning in rats. Physiol. Behav. 70: 351-357.

Rapp, P.R., Rosenberg, R.A., and Gallagher, M. 1987. An evaluation of spatial information processing in aged rats. Behav. Neurosci. 101: $3-12$.

Rosenzweig, E.S. and Barnes, C.A. 2003. Impact of aging on hippocampal function: Plasticity, network dynamics, and cognition. Prog. Neurobiol. 69: 143-179.

Sakamoto, T. and Okaichi, H. 1996. The use of geometrical information and featural information in fimbria-fornix-lesioned rats. Shinrigaku Kenkyu 67: 110-117.

- 1997. The effects of a fimbria-fornix lesion on distance discrimination in rats. Shinrigaku Kenkyu 68: 38-42.

Schulz, D., Huston, J.P., Jezek, K., Haas, H.L., Roth-Harer, A., Selbach, O., and Luhmann, H.J. 2002. Water maze performance, exploratory activity, inhibitory avoidance and hippocampal plasticity in aged superior and inferior learners. Eur. J. Neurosci. 16: 2175-2185.

Sharps, M.J. and Gollin, E.S. 1987. Memory for object locations in young and elderly adults. J. Gerontol. 42: 336-341.

Shors, T.J. 2006. Stressful experience and learning across the lifespan. Annu. Rev. Psychol. 57: 55-85.

Sovrano, V.A., Bisazza, A., and Vallortigara, G. 2002. Modularity and spatial reorientation in a simple mind: Encoding of geometric and nongeometric properties of a spatial environment by fish. Cognition 85: B51-B59.

. 2003. Modularity as a fish (Xenotoca eiseni) views it: Conjoining geometric and nongeometric information for spatial reorientation. $J$. Exp. Psychol. Anim. Behav. Process. 29: 199-210.

. 2005. Animals' use of landmarks and metric information to reorient: Effects of the size of the experimental space. Cognition 97: 121-133.

Tommasi, L., Gagliardo, A., Andrew, R.J., and Vallortigara, G. 2003. Separate processing mechanisms for encoding of geometric and landmark information in the avian hippocampus. Eur. J. Neurosci. 17: $1695-1702$.

Uttl, B. and Graf, P. 1993. Episodic spatial memory in adulthood. Psychol. Aging 8: 257-273.

Vallortigara, G., Zanforlin, M., and Pasti, G. 1990. Geometric modules in animals' spatial representations: A test with chicks (Gallus gallus domesticus). J. Comp. Psychol. 104: 248-254.

Vallortigara, G., Feruglio, M., and Sovrano, V.A. 2005. Reorientation by geometric and landmark information in environments of different size. Dev. Sci. 8: 393-401.

Vargas, J.P., Petruso, E.J., and Bingman, V.P. 2004. Hippocampal formation is required for geometric navigation in pigeons. Eur. J. Neurosci. 20: 1937-1944.

Wall, P.L., Botly, L.C., Black, C.K., and Shettleworth, S.J. 2004. The geometric module in the rat: Independence of shape and feature learning in a food finding task. Learn. Behav. 32: 289-298.

Whishaw, I.Q., Metz, G.A., Kolb, B., and Pellis, S.M. 2001. Accelerated nervous system development contributes to behavioral efficiency in the laboratory mouse: A behavioral review and theoretical proposal. Dev. Psychobiol. 39: 151-170.

Received November 10, 2005; accepted in revised form April 6, 2006. 


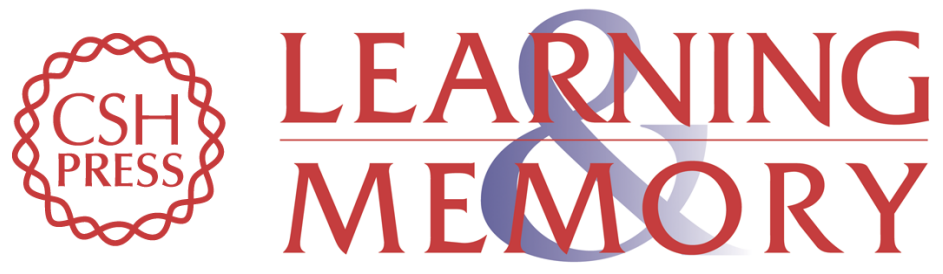

\section{Adult but not aged C57BL/6 male mice are capable of using geometry for orientation}

Laetitia Fellini, Melitta Schachner and Fabio Morellini

Learn. Mem. 2006, 13:

Access the most recent version at doi:10.1101//m.259206

References This article cites 46 articles, 6 of which can be accessed free at:

http://learnmem.cshlp.org/content/13/4/473.full.html\#ref-list-1

License

Email Alerting Receive free email alerts when new articles cite this article - sign up in the box at the Service top right corner of the article or click here. 